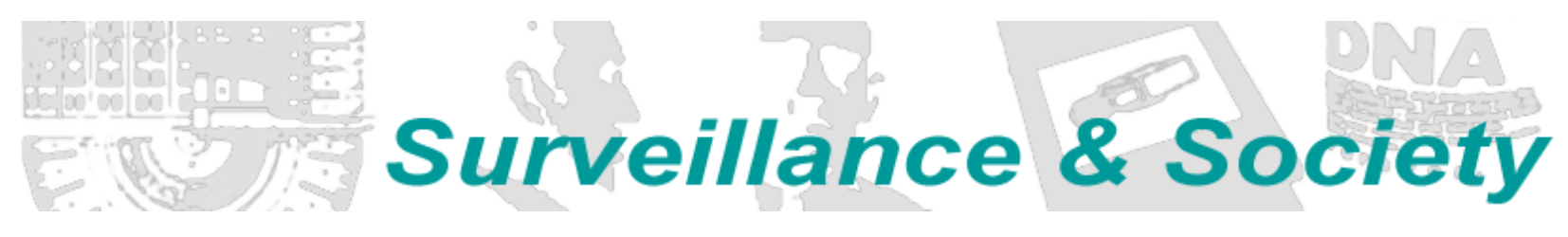

\title{
Essay $\quad$ APAIC Report on the Holocode Crisis
}

\section{Gabriele de Seta}

University of Bergen, Norway

gabriele.seta@uib.no

Re: Mesh Intelligence Transparency Act Request No. 04-MIT-03296-11

\section{APAIC REPORT ON THE HOLOCODE CRISIS}

Redacted extracts of fieldnotes and interview transcripts from the PARGC-funded project \#204122 "A Sensociology of Holocodes: Technoregional Sales of Panspectral Sensing” (PI: Prof. Zhang Ge, ERB Clearance No. 00972GF)

June 22, 2043

Guangzhou-Shengang bullet train $(22.819,113.6731)$

Scheduling a meeting with $\mathrm{Xu} \mathrm{Chia}^{1}$ was smoother than I expected. As a matter of fact, pretty much all research activity related to holocodes has been way smoother once I gave in to registering my biopsymetrics with the local convenience store and consenting to panspectral tracking within the Guangdong jurisdiction for the remaining week of fieldwork. In a matter of minutes, intrusive notifications from both Wanwu and $\mathrm{A}+$ offered me 5,000RMB vouchers in exchange for exclusive access to my public and commercial vectors, which was a welcome surprise (I picked the Wanwu one since the company is based around here and service coverage should be better). Less than half of the sum paid for the cab ride and a massive bowl of Lanzhou Noodles I got on the way - a nostalgic reminiscence of my first research trips here, decades before the franchise became a showcase for the synthetic restoration industry.

The meeting location was chosen by Xu themself, and I got to the lobby of the almost half an hour in advance. Xu Chia was already there and had made sure I was well-prepared for the interview, probably hoping I would avoid asking the usual introductory questions: a drip-feed of biographical snippets kept scrolling down from the top of my disp inside notification frames sporting the familiar blooms of voxellike particles, each message prompted by one of the thousands of sensors tracking my passage through the metropolitan mesh. The only new bit of information among the many was a note about their peculiar first name:

\footnotetext{
1 [Editor's note: Given their public persona and ongoing disappearance, Xu Chia's name has been de-anonymized for the interest of the current inquiry - the original fieldnotes describing this encounter referred to them by the pseudonym "MC"] 
ChiaWare Trivia \#4: The name Chia, which can’t be written with any Chinese character, was chosen by Xu's parents as an auspicious tribute to the homonymous SSD-mined cryptocurrency popular in the early 2020 s.

I think I vaguely remember hearing about this specific craze back then, but I might be wrong [CHECK FIELDNOTE BACKUP ARCHIVE].

Sitting at a long table in one of the business meeting rooms behind the lobby, Xu Chia struck me as the prototypical poster-person of post-rejuvenation China: endearingly self-confident, performatively informal, productively skeptical. Their assistant introduced himself as —_ handed me a textured ChiaWare leaflet featuring the official biographical blurb of its founder, and pointed me to a perfectly arranged line of glazed cups filled with hot water and tea leaves of progressively darker hues as they left the room. "Just call me Chia" was the first thing Xu told me before taking a sip of a colorful drink from a cartoonish, oversized mug with a handle shaped like a pixelated pickaxe. I sat at the empty chair near the two consent forms they had already signed and waited for them to focus out of their disp, checking recording volume levels, rehearsing my list of questions, and staring blankly at the corporate leaflet.

[Transcribed by LarynxType Pro, 08:42/495 words, regular inflection (pensive), sore throat]

[MISSING RESOURCE: IMG_2811; OCR leaflet transcription attached]

$\mathrm{Xu}$ Chia, $\mathrm{PhD}$, is the inventor of holographic codes and founder of ChiaWare Inc., the Chinese multinational technology conglomerate providing granular sensing infrastructure to more than 160 countries.

As a child, Xu Chia demonstrated an analytical mind and an obsession for detail that only early virtual worlds could satisfy. At only fifteen years of age, they completed the first prototype of QuanXiMa, which still lies at the core of the QXM standard decoder utilized across the globe.

In 1947, American inventor Joe Woodland traced the lines of the first barcode on the sand of Miami Beach; in 1994, Japanese inventor Masahiro Hara modeled the QR code on the game of go; in 2036, Xu Chia built the first holocode inside the classic videogame Minecraft, inspired by its iconic voxel architecture.

Growing up under tight restrictions on student use of multiplayer sensoreality, Xu Chia found refuge in the blocky world of Minecraft, spending months building their own personal server. One day, looking at the fading horizon from the top of a floating mountain, it occurred to them that the cubic voxels could be used to encode large amounts of information in three-dimensional space.

Thanks to developments in holographic technology, Xu Chia could miniaturize their virtual space of information into smaller and smaller surfaces: the holocode was born. And thanks to developments in sensor resolution, holocodes would become the most reliable way to share information across supranational meshes.

$\mathrm{Xu}$ Chia founded ChiaWare in 2038, and the QXM national standard supported China's industry 5.0 development, enabling more responsive logistics, more trustworthy automation, and safer consumption. Thanks to China's primacy in exportoriented goods manufacturing, QXM reached all corners of the world, laying the groundwork for holocodes to become the first truly global Chinese standard. 
Q: So, I just started recording our chat as we agreed. Thanks for making the time for a brief interview, this is a key meeting for my research project, as you can imagine.

A: Well... thank you, thank you. It is always a pleasure to talk to someone interested in quanxima, ${ }^{2}$ most people are obsessed with the latest developments in sensoreality or love speculating about next year's disp trends, while they find encodings and standards to be a boring topic.

Q: That's very true! We're the minority here. Well, I guess you already know all you need to know about me... actually, maybe, we could start from this: how much do you know about me, and how exactly has information about me reached you?

A: [Laughter.] I'm afraid this might disappoint you, but this is the first question that almost every interviewer asks me, and the answer is "very little." I know what my outreach assistant told me, which is how you introduced yourself via mail. I didn't have time to look you up, so they compiled a brief memo for me. A "sensociologist," if I understand correctly, currently working on a research project about technoregionalism? That's pretty much all I know. It is a common misconception that I have some sort of personal access to our users' information. I do not, and ChiaWare does not have it either. I have patented QXM in 2037-I'm sure you are already familiar with the whole history - and my main goal was to automate the sharing of information between different technological layers so that humans could be kept safe by being kept out of the loop. So yes, the many quanxima etched on the rims of your disp, on your wristie, on your sneaker soles and your CityToken all contain vast amounts of sensor-readable information about both you and the objects themselves, but the use that others can make of this information is determined by contractual agreements that I'm sure you have learned to negotiate. On your way here...

Q: I did. I got a 5,000RMB voucher from Wanwu, seemed like the best deal for lunch. Sorry to interrupt you, go on.

A: I would have picked A+ myself. Guangdong is Wanwu's home turf, it's always safer to bet on the outsider, which has to work harder in order to remain competitive. But you're not alone, I noticed that many users cling to their regional allegiances as a form of consumer identity... this is something sociologists have written about, right? We have successfully become the New People's Republic of China, but these "new people" are still a work-in-progress. Anyway... on your way here, I was saying, you received several messages from me, is that right? Those were triggered by some general conditions I set, like your distance from here and the time left before your arrival. Various federated systems relied on public and private sensors to trace your movements and deliver the messages at the appropriate moment. As a matter of fact, sensors on your own devices also helped triangulate your position whenever they scanned the quanxima embedded on people and objects around you. It's a constant exchange of information about the status, position, and configuration of objects in space and time, but the use that every actor can make of the sensed information is strictly regulated by mesh-specific relational contracts.

Q: So, there is no back-end, no control center, no dashboard? How does ChiaWare deal with security?

A: None of that. We barely have access to information about our own assets. You can't imagine how fiddly it can be even just to locate a company EV if an employee parks it in the wrong spot and forgets to release the contract for the weekend. Privacy regulation has come a long way.

Q: But couldn't someone with a QXM decoder use it to unlawfully retrieve and collect information about objects and people around them?

2 The Chinese name of holocodes is quanxima, sometimes abbreviated as QXM, which literally translates as "fullinformation code" or "holographic code." 
A: Not really. Our own commercial sensors as well as third-party ones are hardcoded to read only specific layers of information, and to output selected values depending on what contracts between public and private entities request of them. That's an integral feature of the QXM standard. Everything happens inside the mesh, and every exchange is encrypted. That's the beauty of holograms - they can contain nearly infinite amounts of enfolded information, but no single actor is allowed to access it all. All users can do is establish relational contracts between one another and formulate specific triggers to exchange information under temporally bound circumstances. What we provide is an overlay of passive informational beacons, a shifting matrix of bottomless data wells... I like to explain it through a metaphor from Buddhism, the "Flower Garland." Have you ever heard of it? Every holocode contains "a realm in a point the size of a hairtip," as the sutra says. Quanxima are still quite larger than a hairtip, but that's the best we can do with today's holographic etching [laughter].

Q: Got it. I understand this question might sound confrontational, but I am genuinely curious about your response to a certain line of critique: theoretically speaking, at least you, the person with access to the original QXM model, could get to the bottom of these "data wells" or decode more information than any relational contract allows....

A: Oh, I see you've done your research on conspiracy theories as well. You probably know these are spun out of rumors circulated by our less and less relevant Euro-American competitors. I will repeat ChiaWare's main value proposition: I do not have access to the original model anymore. Every single holocode is procedurally generated through an encryption algorithm derived from the original model I coded as a kid. My parents threw away that laptop long ago, Minecraft server and all, without knowing what it contained; all I have left is algorithms to generate new holocodes and relational contracts to decrypt selected sections of them. This is publicly documented and functions as the fundamental proof of holocode robustness - they are locked with a key that doesn't exist anymore. The hard drive where the model was stored has very likely been formatted or scrapped for raw materials, and even if someone found that specific Minecraft save file, they would have no clue what they were looking at. What at the time I thought was a setback turned out to be the key to our standard's success.

Q: Please remember that this is being recorded, but you'll be able to request that specific sections to be erased at any point in the future. And we can go off the record if you prefer.

A: Absolutely no need. If I really had any form of privileged access, I might just decide to trace your disp and send someone to wipe out your archive, right? [Laughter.] You can write about all of this - even if it only serves to prove how confident I am about the security of our product.

[Relevant excerpt ends here-Full interview transcript available in the PARGC repository]

\section{Provisional conclusions from the APAIC investigation on the disappearance of NPRC citizen XU Chia October 21, 2046}

As concluded by the task force appointed to trace back the movements of Dr. XU Chia of ChiaWare Inc., following their exit from China' borders in late April 2046, their disappearance is likely to be related to information contained in the excerpts compiled in this report.

Extensive intelligence work has confirmed with high confidence the following timeline: having left Guangzhou on a chartered flight for a brief stopover in Singapore, Dr. XU boarded a private suborbital vehicle directed to the Maputo spaceport.

According to leaks of internal corporate communications, the Mozambique visit was scheduled as the beginning of a series of business visits to African countries interested in expanding their adoption of ChiaWare sensor technologies. 
It is of note that, through the long-standing involvement of China in Mozambique's mesh infrastructurebuilding, the country had already reached a sensor coverage of $84.5 \%$ in 2044 . This discrepancy with other planned destinations casts doubts on the actual purpose of Dr. XU's visit.

Interviews on the ground seem to point to a more likely explanation: the resurfacing in Mozambique of an item of interest for Dr. XU-namely, the laptop (or at least its solid-state drive) containing the original QXM model (henceforth: the Device).

It is now believed that, in their teenage years, Dr. XU Chia had etched early prototypes of holocodes on individual components of the Device containing the original model before this was disposed of by their parents, betting on the success of their invention and on its eventual traceability.

This future-oriented failsafe mechanism became operational once QXM succeeded as a global standard, and sensors from the Mozambique mesh eventually identified the holocodes of the Device (or of its components) as they passed through the local e-waste recycling industry.

Unverified reports point to Mr. XU being spotted in Xai-Xai District, a key hub of e-waste processing, in early May 2046. No further testimonies are available, and the likelihood of Mr. XU being currently in possession of the original holocode model is now being evaluated by sensorsecurity agencies worldwide.

No conclusion has been reached regarding Dr. XU's actual capability of fully decoding QXM-encoded data. While several supranational mesh authorities are scrambling to adopt alternatives and set up redundancies in what has been popularly termed the "Holocode Crisis," the security implications resulting from the threat of absolute legibility remain unclear.

\section{Research Statement}

The APAIC Report on the Holocode Crisis is a short story that imagines the future of machine-readable data encodings. In this story, I speculate about the next stage in the development of data encoding patterns: after barcodes and QR codes, the invention of "holocodes" will make it possible to store unprecedented amounts of data in a minuscule physical surface. As a collage of nested fictional materials (including ethnographic fieldnotes, interview transcripts, OCR scans, and intelligence reports) this story builds on the historical role of barcodes in supporting consumer logistics and the ongoing deployment of QR codes as anchors for the platform economy, concluding that the geopolitical future of optical governance (de Seta 2020) is tied to unassuming technical standards such as those formalizing machine-readable representations of data. More broadly, the APAIC Report is a meditation on traceability; by describing a world in which most human and non-human actors are constantly immersed in a mesh of optical sensors and encoded data, I explore one of the many ways in which the dream of "making all knowledge retrievable" (Bowker and Star 1999: 24) can fail once it is put into practice. My depiction of events happening two decades in the future is directly related to the sort of "comprehensive monitoring associated with the proliferation of distributed, embedded, alwayson sensing network" that Mark Andrejevic (2019: 7) has identified as a result of the automation of surveillance. Making the world machine-readable through classification and standardization is a key component of this process, and supply chains play a central role in the infrastructural entrenchment of data encoding formats, contributing to the creation of a planetary surveillable space (Archer 2021). The APAIC Report on the Holocode Crisis depicts a hypothetical surveillance future at a threshold of uncertainty and possibility, leaving open questions about our current relationships to data encodings and machine-readable artifacts. 


\section{Funding}

This research is supported by the Machine Vision in Everyday Life project, which has received funding from the European Research Council (ERC) under the European Union's Horizon 2020 research and innovation programme (Grant Agreement No. 771800).

\section{Acknowledgments}

Thanks to Samantha Culp, Jill Walker Rettberg, and En-ping Yu for reading early drafts of this story and offering helpful comments and suggestions.

\section{References}

Andrejevic, Mark. 2019. Automating Surveillance. Surveillance \& Society 17 (1-2): 7-13.

Archer, Matthew. 2021. Imagining Impact in Global Supply Chains: Data-Driven Sustainability and the Production of Surveillable Space. Surveillance \& Society 19 (3): 282-298.

Bowker, Geoffrey C., and Susan Leigh Star. 1999. Sorting Things Out: Classification and Its Consequences. Cambridge, MA: MIT Press.

de Seta, Gabriele. 2020. Optical Governance: The Roles of Machine Vision in China's Epidemic Response. Strelka Mag, October 11. https://strelkamag.com/en/article/optical-governance [accessed November 27, 2021]. 